\title{
Prévalence de Cryptosporidium spp. et Giardia spp. chez les bovins de la région de Sétif au nord-est de l'Algérie
}

\author{
N. Ouchene ${ }^{1 *}$ N.A. Ouchene-Khelifi ${ }^{1}$ \\ M. Aissi ${ }^{2}$ A. Benakhla ${ }^{1}$
}

Mots-clés

Bovin - Veau - Cryptosporidium Giardia - Morbidité - Diarrhée Algérie.

\begin{abstract}
Résumé
La prévalence de Cryptosporidium spp. et de Giardia spp. a été étudiée dans 13 élevages bovins laitiers de la région de Sétif au nord-est de l'Algérie. Au total, 634 prélèvements de fèces ont été réalisés dont 302 chez des veaux et 332 chez des adultes. Cryptosporidium spp. a été identifié dans neuf élevages (69,2 p. 100) et dans 22 p. 100 de l'ensemble des animaux. Le parasite a été observé dans toutes les classes d'âge avec une prévalence plus élevée chez les veaux de 4 à 30 jours $(p<0,01)$. L'excrétion d'oocystes de Cryptosporidium spp. a été très significativement associée aux diarrhées, notamment chez les veaux âgés de moins de 30 jours $(p<0,001)$. D'autre part, Giardia spp. a été identifié dans 53,8 p. 100 des élevages et chez 13,8 p. 100 de l'ensemble des animaux. Contrairement à Cryptosporidium spp., I'infestation par Giardia spp. a semblé plus élevée chez les veaux plus âgés $(4-12$ mois) $(p<0,05)$. Aucune association significative n'a été signalée entre l'excrétion des kystes de Giardia spp. et le type des fèces. L'association des deux parasites a été trouvée chez 10,2 p. 100 de l'ensemble des veaux diarrhéiques. Ce travail montre I'importance de Cryptosporidium spp. et, pour la première fois en Algérie, de Giardia spp. chez les bovins laitiers de la région de Sétif.
\end{abstract}

\section{INTRODUCTION}

Cryptosporidium spp. est un protozoaire parasite des voies digestives de nombreuses espèces animales et de l'homme (15). Deux espèces de Cryptosporidium spp. sont rencontrées chez les bovins : Cryptosporidium parvum à localisation surtout intestinale, qui est l'espèce la plus fréquente chez les jeunes et la plus pathogène (20), et $C$. andersoni, parasite de la caillette des bovins adultes, rarement pathogène (7). C. parvum a longtemps été considéré comme un agent de surinfection, jusqu'aux années 1970 où il fut responsable d'épidémies de diarrhées néonatales, parfois mortelles, dans les élevages des jeunes veaux (18). Durant les deux premières semaines de vie, le veau peut excréter des milliers d'oocystes de

1. Institut des sciences vétérinaires, Université d'El-Tarf, BP 73, El-Tarf 36000, Algérie.

2. Ecole nationale supérieure vétérinaire, El-Harrach, Alger, Algérie.

* Auteur pour la correspondance

Tél. : +213661224250 ; e-mail : ouchenassim@gmail.com
C. parvum (25), alors que les individus adultes restent la plupart du temps des porteurs asymptomatiques (20).

Giardia spp. est un protozoaire parasite des bovins, de l'homme et d'autres vertébrés, transmis par voie oro-fécale (1). Chez les bovins, G. intestinalis (synonymes G. duodenalis ou G. lamblia) peut provoquer des diarrhées, une malabsorption des nutriments et des retards de croissance (19). L'élimination des kystes dans les fèces peut durer plusieurs semaines (26). Contrairement à $C$. parvum, $G$. intestinalis affecte les veaux les plus âgés avec une tendance à la chronicité (16).

En Algérie, puisque, à ce jour, les informations concernant l'épidémiologie de la cryptosporidiose bovine restent toujours limitées et aucune enquête épidémiologique n'a été réalisée sur la giardiose bovine, il est apparu intéressant de participer à l'étude de la cryptosporidiose et de la giardiose chez l'espèce bovine. L'objectif du présent travail a été de déterminer la prévalence d'excrétion de Cryptosporidium spp. et de Giardia spp. chez les veaux et chez les adultes dans 13 élevages situés dans la région de Sétif au nord-est de l'Algérie. 


\section{MATERIEL ET METHODES}

\section{Prélèvements}

L'étude a été réalisée entre juillet 2006 et avril 2007 dans 13 élevages bovins laitiers dont quatre étatiques (race Prim'Holstein) et neuf privés (race Prim'Holstein et Montbéliarde). Dans tous ces élevages et après la naissance, les veaux restaient auprès de leur mère jusqu'à l'âge de deux semaines et étaient ensuite isolés dans des box collectifs. Ainsi, les diarrhées ont été observées chaque année et aucune prévention ou traitement n'a été entrepris contre la cryptosporidiose. Un total de 634 prélèvements de matières fécales ont été réalisés dont 302 issus de veaux (âgés d'un jour à 12 mois) et 332 issus de vaches. Chaque animal a fait l'objet d'un seul prélèvement. Ces prélèvements ont été recueillis directement à partir du rectum dans des flacons stériles puis acheminés dans une glacière au laboratoire de parasitologie de l'Ecole nationale supérieure vétérinaire d'Alger où ils ont été stockés pendant trois jours au maximum à une température de $4{ }^{\circ} \mathrm{C}$ en vue d'une recherche ultérieure des parasites ciblés.

\section{Analyse de laboratoire}

Pour la recherche de Giardia spp., chaque prélèvement a été analysé par la technique de concentration de Ritchie simplifiée par Allen et Ridley (3) : une noisette de matière fécale a été mélangée avec $7 \mathrm{ml}$ de formol à 10 p. 100, filtrée sur gaze, $3 \mathrm{ml}$ d'éther ont été ajoutés, le tout a été agité vigoureusement et centrifugé à $1600 \mathrm{~g}$ pendant $1 \mathrm{~min}$. Une goute du culot de centrifugation a été posée entre lame et lamelle pour la recherche de Giardia spp. L'examen a eu lieu sous microscope optique $(\times 200$ et $\times 400)$.

Pour rechercher les oocystes de Cryptosporidium spp., un frottis a été préparé pour chaque prélèvement à partir du culot de centrifugation obtenu par la même technique de concentration de Ritchie simplifiée par Allen et Ridley (3), ensuite le frottis a été coloré par la technique de coloration de Ziehl-Neelsen modifiée par Henriksen et Pohlenz (9). L'examen a eu lieu sous microscope optique $(\times 400$ et $\times 800)$.

\section{Analyses statistiques}

L'étude de l'influence de l'âge et du type de matières fécales sur la prévalence d'excrétion de Cryptosporidium spp. et de Giardia spp. et l'association entre les deux parasites, a été réalisée par le test $\chi^{2}$ (résultats significatifs lorsque $\mathrm{p}<0,05$ )

\section{Tableau I}

Prévalence de I'excrétion de Cryptosporidium spp. et de Giardia spp. en fonction de l'âge des bovins

\begin{tabular}{lrcc}
\multicolumn{5}{c}{ Pourcentage d'animaux infestés } \\
Age & $\begin{array}{c}\text { Nb. de } \\
\text { prélèvements }\end{array}$ & Cryptosporidium spp. & Giardia spp. \\
\hline 1-3 jours & 8 & 0 & 0 \\
4-14 jours & 31 & 41,9 & 9,6 \\
15-30 jours & 51 & 39,2 & 13,7 \\
1-3 mois & 94 & 23,4 & 13,8 \\
4-12 mois & 118 & 18,6 & 21,2 \\
Adultes & 332 & 18,9 & 12 \\
\end{tabular}

\section{RESULTATS}

A l'issue de cette enquête, 69,2 p. 100 (9/13) des élevages possédaient au moins un veau présentant des oocystes de Cryptosporidium spp. et, sur un effectif de 634 bovins, 140 (22 p. 100) excrétaient des oocystes de Cryptosporidium spp. Par rapport à l'âge, la prévalence d'une infestation par les cryptosporidies s'est révélée plus élevée chez les veaux de 4 à 30 jours que chez les veaux de plus de 30 jours $(\mathrm{p}<0,01)$. Les oocystes ont été observés dans les fèces des animaux à partir du quatrième jour de naissance (tableau I). Les kystes de Giardia spp. ont été observés dans 53,8 p. 100 (7/13) des élevages et chez 13,8 p. 100 des 634 bovins étudiés, et ce, à partir du huitième jour d'âge jusqu'à l'âge adulte (tableau I). Les veaux âgés de 4 à 12 mois ont été les plus infestés (21,2 p. 100) par rapport aux autres classes d'âge $(\mathrm{p}<0,05)$.

Par ailleurs, les adultes ont excrété des oocystes de Cryptosporidium spp. de manière asymptomatique : pas de diarrhées signalées chez eux. En revanche, chez les 302 veaux (âgés de 1 jour à 12 mois), l'excrétion d'oocystes de Cryptosporidium spp. a semblé très significativement liée à la présence de diarrhées $(\mathrm{p}<0,001)$. Des oocystes ont été mis en évidence chez 65,3 p. 100 des veaux diarrhéiques et 17,8 p. 100 des veaux non diarrhéiques (tableau II). Les veaux âgés de 4 à 30 jours ont été les plus concernés par des diarrhées avec une prévalence de 84,6 p. 100 (p < 0,001), contre 43,6 p. 100 chez ceux âgés d'un jour à 12 mois. Par ailleurs, l'étude statistique n'a révélé aucune association significative entre l'état des fèces et la prévalence d'excrétion de kystes de Giardia spp. (tableau II).

Parmi les 49 veaux diarrhéiques, 27 (55,1 p. 100) ont montré des oocystes de Cryptosporidium spp. sans présence de kystes de Giardia spp. Cette prévalence a été très significativement élevée par rapport à celle de l'excrétion de kystes de Giardia spp. seuls ou en association avec les oocystes de Cryptosporidium spp. ( $<<0,001)$ (tableau III).

\section{Tableau II}

Prévalence de l'excrétion de Cryptosporidium spp. et de Giardia spp. en fonction de l'état des fèces chez les veaux âgés de 1 jour à 12 mois

\begin{tabular}{lcc|}
\hline & \multicolumn{2}{c}{ Nb. de veaux infestés (\%) } \\
\cline { 2 - 3 } & Cryptosporidium spp. & Giardia spp. \\
\hline $\begin{array}{l}\text { Fèces diarrhéiques } \\
(n=49)\end{array}$ & $32(65,3)$ & $17(34,8)$ \\
$\begin{array}{l}\text { Fèces non diarrhéiques } \\
(n=253)\end{array}$ & $45(17,8)$ & $71(28)$ \\
& & \\
\end{tabular}

\section{Tableau III}

Prévalence de l'excrétion chez les veaux diarrhéiques âgés de 1 jour à 12 mois et des deux parasites seuls ou associés

Nb. de veaux infestés sur 49 (\%)

$\begin{array}{lc}\text { Cryptosporidium spp. seul } & 27(55,1) \\ \text { Giardia spp. seul } & 3(6,1) \\ \text { Cryptosporidium spp. + Giardia spp. } & 5(10,2)\end{array}$




\section{DISCUSSION}

A Sétif, l'infestation des bovins par les deux parasites a semblé très fréquente. Ainsi, 69,2 et 53,8 p. 100 des élevages possédaient au moins un bovin présentant respectivement des oocystes de Cryptosporidium spp. ou des kystes de Giardia spp. Ces proportions sont proches de celles signalées par Quílez et coll. (19) qui rapportent 63,3 p. 100 de fermes infestées par Cryptosporidium spp. et 53,3 par Giardia spp. De leur côté, Ruest et coll. (21) rapportent 88,7 p. 100 de fermes infestées par Cryptosporidium spp. et 45,7 p. 100 par Giardia spp.

Sur les 634 bovins de la présente étude, 22 p. 100 ont été infestés par des oocystes de Cryptosporidium spp. avec une prévalence de 25,5 p. 100 chez les veaux âgés d'un jour à 12 mois et 18,9 p. 100 chez les adultes. Ces prévalences sont proches de celles signalées par plusieurs auteurs chez les veaux comme chez les adultes (11, 13, 19).

L'infestation des veaux par les oocystes de Cryptosporidium spp. peut se faire dès la naissance et l'élimination fécale des oocystes commence au quatrième jour post-partum (14). Dans la présente étude, aucun veau âgé de moins de quatre jours n'a excrété d'oocystes de Cryptosporidium spp., et cette observation a été identique à celles rapportées par Quílez et coll. (19), et Kvac et coll. (12).

La cryptosporidiose est une maladie du jeune animal, elle se manifeste principalement chez les veaux âgés de 4 à 30 jours (2). Dans les présents résultats, la prévalence la plus élevée a été observée chez les veaux âgés de 4 à 30 jours $(\mathrm{p}<0,01)$, avec un pic chez les veaux de 4 à 14 jours, comme observé par d'autres auteurs $(10,11$, 19, 23).

Durant les deux premières semaines de vie, le veau peut excréter des milliers d'oocystes de $C$. parvum (25), alors que les individus adultes restent la plupart du temps des porteurs asymptomatiques (20). Dans le présent travail, les adultes se sont révélés excréteurs asymptomatiques d'oocystes de Cryptosporidium spp. et aucun animal n'a été signalé comme présentant des diarrhées. La concentration des prélèvements (technique de Ritchie) permet de détecter les oocystes dans les fèces, même à un nombre faible, comme cela a été le cas chez les adultes de l'étude. Toutefois, la prévalence relativement élevée (18,9 p. 100) de l'infestation chez les adultes était due au fait que cette catégorie comprenait seulement des vaches venant de vêler, et ce, dans un milieu très contaminé par les cryptosporidies. La même remarque a été faite par d'autres auteurs $(6,10,17,19)$.

Plusieurs auteurs ont signalé une prévalence plus élevée du parasite dans les fèces d'animaux diarrhéiques $(5,10,13,25)$. De même dans la présente étude, Cryptosporidium spp. a été davantage rencontré chez les veaux diarrhéiques que chez ceux qui ne l'étaient pas, et surtout chez ceux âgés de moins de 30 jours $(\mathrm{p}<0,001)$. Durant les premiers jours de vie, le système immunitaire des veaux est encore immature, d'où cette grande sensibilité à l'infection cryptosporidienne (14).

Concernant Giardia spp., les résultats de la présente étude ont révélé une prévalence de 13,8 p. 100 sur l'ensemble des bovins et les kystes ont été observés dans toutes les classes d'âge. Ces résultats sont proches de ceux rapportés par Quílez et coll. (19) avec une prévalence de 11,7 p. 100 et l'excrétion de kystes de Giardia spp. observée dans toutes les classes d'âge.

A la différence de $C$. parvum très présent chez les veaux de moins de 30 jours, G. duodenalis affecte des veaux plus âgés avec une tendance à la chronicité (16). L'âge de l'excrétion des kystes peut commencer au quatrième jour après la naissance (26) mais la période prépatente a été évaluée à 7-8 jours chez des veaux infestés expérimentalement (24). Dans le présent travail, l'excrétion des kystes de Giardia spp. a commencé dès l'âge de 8 jours avec la prévalence la plus élevée chez les veaux âgés de 4 à 12 mois ( $\mathrm{p}<0,05)$. Cependant, beaucoup d'études restent controversées sur cette question. Une étude aux Pays-Bas a montré que la prévalence la plus élevée était observée chez les veaux de 4-5 mois (10). De même, une étude menée en Colombie britannique a indiqué que 80 p. 100 des veaux âgés de 2 à 24 semaines étaient infestés par G. duodenalis (17). Quílez et coll. (19) rapportent que la prévalence d'excrétion maximale des kystes se situe entre l'âge de 1,5 et 4 mois.

Giardia spp. peut être à l'origine de diarrhées chez les veaux (27), mais il existe des excréteurs asymptomatiques (19). Dans le présent travail, la différence entre veaux diarrhéiques et veaux non diarrhéiques excrétant des kystes de Giardia spp. n'a pas été significative. Ces résultats sont comparables à ceux observés par Quílez et coll. (19), et Huetink et coll. (10). G. duodenalis, seul, peut causer des diarrhées chez les bovins mais il peut aussi être associé à Cryptosporidium spp. (22). De nombreuses études ont montré une concurrence entre l'infestation par G. duodenalis et Cryptosporidium spp. chez les veaux $(4,8,21)$.

\section{CONCLUSION}

Cryptosporidium spp. et Giardia spp. ont été très fréquemment rencontrés dans les élevages bovins laitiers de la région de Sétif. Les deux parasites ont été isolés dans toutes les classes d'âge avec une prédominance de Cryptosporidium spp. chez les veaux de 4 à 30 jours et de Giardia spp. chez les veaux de 4 à 12 mois. A la différence de Giardia spp., l'excrétion d'oocystes de Cryptosporidium $\mathrm{spp}$. a semblé très liée aux diarrhées, notamment chez les veaux âgés de 4 à 30 jours. Cela indique l'importance du rôle de la cryptosporidiose dans les diarrhées néonatales des veaux simultanément à d'autres agents pathogènes (rotavirus, coronavirus et Escherichia coli) (5). Ainsi, l'association des deux parasites chez les veaux diarrhéiques est possible mais ces derniers ont semblé davantage infestés par Cryptosporidium spp. seul. La détection des oocystes de Cryptosporidium spp. dans les fèces des adultes a montré le rôle important de ces derniers comme source d'infestation pour les jeunes veaux.

\section{BIBLIOGRAPHIE}

1. ADAM R.D., 1991. The biology of Giardia spp. Microbiol. mol. Biol. Rev., 55: 706-732.

2. ALAIN V., 2003. Les zoonoses parasitaires : I'infection chez les animaux et chez I'homme. Montréal, Québec, Canada, Presses de I'université de Montréal, $500 \mathrm{p}$.

3. ALLEN V.H., RIDLEY D.S., 1970. Further observations on the formol ether concentration technique for faecal parasites. J. clin. Pathol., 23: 545-546.

4. BJORKMAN C., SVENSSON C., CHRISTENSSON B., DE VERDIER K., 2003. Cryptosporidium parvum and Giardia intestinalis in calf diarrhoea in Sweden. Acta vet. Scand., 44: 145-152.

5. CASTRO-HERMIDA J.A., GONZILEZ-LOSADA Y.A., ARESMAZAS E., 2002. Prevalence and risk factors involved in the spread of neonatal bovine cryptosporidiosis in Galicia (NW Spain). Vet. Parasitol., 106 $1-10$.

6. DARABUS G.H., COSOROABA I., OPRESCU I., MORARIU S., 2001. Epidémiologie de la cryptosporidiose chez les animaux dans l'ouest de la Roumanie. Rev. Méd. vét., 152 : 399-404. 
7. ENEMARK H.L., AHRENS P., LOWERY C.J., THAMSBORG S.M., ENEMARK J.M., BILLE-HANSEN V., LIND P., 2002. Cryptosporidium andersoni from a Danish cattle herd: identification and preliminary characterization. Vet. Parasitol., 107: 37-49.

8. HAMNES I.S., GJERDE B., ROBERTSON L., 2006. Prevalence of Giardia and Cryptosporidium in dairy calves in three areas of Norway. Vet. Parasitol., 140: 204-216.

9. HENRIKSEN S.A., POHLENZ J.F.L., 1981. Staining of cryptosporidia by a modified Ziehl-Neelson technique. Acta vet. Scand., 22: 594-596.

10. HUETINK R.E.C., VAN DER GIESSEN J.W.B., NOORDHUIZEN J.P.T.M., PLOEGER H.W., 2001. Epidemiology of Cryptosporidium spp. and Giardia duodenalis on a dairy farm. Vet. Parasitol., 102: 53-67.

11. KHELEF D., SAIB M.Z., AKAM A., KAIDI R., CHIRILA V., COZMA V., ADJOU K.T., 2007. Epidémiologie de la cryptosporidiose chez les bovins en Algérie. Rev. Méd. vét., $158:$ 260-264.

12. KVAC M., KOUBA M., VITOVEC J., 2006. Age-related and housing dependence of Cryptosporidium infection of calves from dairy and bee herds in South Bohemia, Czech Republic. Vet. Parasitol., 137: 202-209.

13. LEFAY D., NACIRI M., POIRIER P., CHERMETTE R., 2000. Prevalence of Cryptosporidium infection in calves in France. Vet. Parasitol., 89: 1-9.

14. MORIN R., 2002. Lutte contre I'infection à Cryptosporidium parvum : application à la cryptosporidiose bovine. Thèse Doct., Ecole nationale vétérinaire, université de Nantes, France.

15. O'DONOGHUE P.J., 1995. Cryptosporidium and cryptosporidiosis in man and animals. Int. J. Parasitol., 25: 139-195.

16. O'HANDLEY R.M., COCKWILL C., MCALLISTER T.A., JELINSK M., MORCK D.W., OLSON M.E., 1999. Duration of naturally acquired giardiasis and cryptosporidiosis in dairy calves and their association with diarrhea. J. Am. vet. med. Assoc., 214: 391-396.

17. OLSON M.E., GUSELLE N.J., O'HANDLEY R.M., SWIFT M.L., MCALLISTER T.A., JELINSKI M.D., MORCK D.W., 1997. Giardia and Cryptosporidium in dairy calves in British Columbia. Can. vet. J., 38 703-706.

\section{Summary}

Ouchene N., Ouchene-Khelifi N.A., Aissi M., Benakhla A. Prevalence of Cryptosporidium spp. and Giardia spp. in cattle in the Setif region of Northeastern Algeria

The prevalence of Cryptosporidium spp. and Giardia spp. in cattle was studied in 13 dairy farms in the Setif region of Northeastern Algeria. A total of 634 fecal samples were collected from 302 calves and 332 adults. Cryptosporidium spp. was identified in nine farms $(69.2 \%)$ and $22 \%$ of animals. The parasite was observed in all age groups but with a higher prevalence in calves aged $4-30$ days $(p<0.01)$. Cryptosporidium spp. oocyst excretion was highly significantly associated with diarrheas, with a higher prevalence in calves under 30 days of age $(p<0.001)$. Giardia spp. was identified in $53.8 \%$ of farms and $13.8 \%$ of animals. Contrary to Cryptosporidium spp., infection by Giardia spp. seemed higher in older calves (4-12 months) $(p<0.05)$. No significant link was established between Giardia spp. cyst excretion and the type of feces. The presence of both parasites was revealed in $10.2 \%$ of diarrheic calves. This work shows the importance of Cryptosporidium spp. and, for the first time, that of Giardia spp. in dairy cattle of the region of Setif.

Keywords: Cattle - Calf - Cryptosporidium - Giardia Morbidity - Diarrhea - Algeria.
18. PANCIERA R.J., THOMASSEN R.W., GARNER F.M., 1971. Cryptosporidial infection in a calf. Vet. Parasitol., 8: 479-484.

19. QUILEZ J., SANCHEZ-ACEDO C., DEL CACHO E., CLAVEL A., CAUSAPE A.C., 1996. Prevalence of Cryptosporidium and Giardia infections in cattle in Aragon (Northeastern Spain). Vet. Parasitol., 66: $139-146$

20. RAMIREZ N.E., WARD L.A., SREEVATSAN S., 2004. A review of the biology and epidemiology of cryptosporidiosis in humans and animals. Microbes Infect., 6: 773-785.

21. RUEST N., FAUBERT G.M., COUTURE Y., 1998. Prevalence and geographical distribution of Giardia spp. and Cryptosporidium spp. in dairy farms in Quebec. Can. vet. J., 39: 697-700.

22. SNEL S.J., BAKER M.G., KAMALESH V., FRENCH N., LEARMONTH J., 2009. A tale of two parasites: the comparative epidemiology of cryptosporidiosis and giardiasis. Epidemiol. Infect., 137: 1641-1650.

23. SOBEIH M., TACAL J.V., WILCKE B.W., LAWRENCE W., EL-AHRA A., 1987. Investigation of cryptosporidial infection in calves in San Bernardino County, California. J. Am. vet. med. Assoc., 191: 816-818.

24. TAMINELLI V., ECKERT J., SYDLERT T., GOTTSTEING B., CORBOZ L., HOFMAN M., 1989. Experimental infection of calves and lambs with bovine giardia isolates. Schweiz. Arch. Tierheilkd., 131: 551-564.

25. UGA S., MATSUO J., KONO E., KIMURA K., INOUE M., RAI S.K., ONO K., 2000. Prevalence of Cryptosporidium parvum infection and pattern of oocyst shedding in calves in Japan. Vet. Parasitol., 94: 27-32.

26. XIAO L., HERD R.P., 1994. Infection patterns of Cryptosporidium and Giardia in calves. Vet. Parasitol., 55: 257-262.

27. XIAO L., HERD R.P., RINGS D.M., 1993. Concurrent infections of Giardia and Cryptosporidium on two Ohio farms with calf diarrhea. Vet. Parasitol., 51: 41-48.

Accepté le 27.09.2012

\section{Resumen}

Ouchene N., Ouchene-Khelifi N.A., Aissi M., Benakhla A. Prevalencia de Cryptosporidium spp. y de Giardia spp. en bovinos de la región de Sétif, nordeste argelino

Se estudió la prevalencia de Cryptosporidium spp. y de Giardia spp. en 13 crías de bovinos lecheros de la región de Sétif, al nordeste de Argelia. Se obtuvieron 634 muestras de heces en total, de las cuales 302 en terneros y 332 en adultos. Cryptosporidium spp. se identificó en nueve establecimientos $(69.2 \%)$ y en $22 \%$ del conjunto de los animales. El parásito se observó en todas las clases de edad, con una prevalencia más elevadas en los terneros de 4 a 30 días $(p<0,01)$. La excreción de oocitos de Cryptosporidium spp., fue significativamente asociada a las diarreas, sobre todo en los terneros de menos de 30 días de edad $(\mathrm{p}<0,01)$. Giardia spp. fue identificado en $53.8 \%$ de los establecimientos y en $13.8 \%$ del conjunto de los animales. Contrariamente a Cryptosporidium spp., la infección de Giardia spp. pareció más elevada en los terneros de mayor edad $(4-12$ mois $)(p<0,05)$. Ninguna asociación significativa se indicó entre la excreción de quistes de Giargia spp. y el tipo de heces. La asociación de los dos parásitos se encontró en 10,2\% del conjunto de los terneros diarreicos. Este estudio muestra la importancia de Cryptosporidium spp. y por la primera vez en Argelia, de Giardia spp. en los bovinos lecheros de la region de Setif.

Palabras clave: Ganado bovino - Ternero - Cryptosporidium Giardia - Morbosidad - Diarrea - Argelia. 